\title{
Synchronized Oscillations of Arterial Oxygen Saturation, Cerebral Tissue Oxygenation and Heart Rate in Preterm Neonates: Investigation of Long-Term Measurements with Multiple Einstein's Cross Wavelet Analysis
}

\author{
Scholkmann, Felix ; Herrera, Graciela Velasco ; Karen, Tanja ; Isler, Helene ; Ostojic, Daniel ; Herrera,
} Victor Manuel Velasco ; Wolf, Ursula; Wolf, Martin

\begin{abstract}
BACKGROUND: In preterm neonates, the cardiovascular and cerebral vascular control is immature, making the brain vulnerable to an increased incidence of hypoxic and hyperoxic episodes. AIM: The aim of the study was to apply the recently developed multiple Einstein's cross wavelet analysis (MECWA) to quantify the coupling of fluctuations of peripherally measured arterial oxygen saturation (SpO2), cerebral tissue oxygen saturation (StO2) and heart rate (HR). METHODS: Two long-term measurements on preterm neonates with a gestational age at birth of 26.4 and 26.8 weeks and a postnatal age of 2.1 and 3.9 weeks were analyzed. MECWA was applied to SpO2, StO2 and HR. RESULTS: MECWA showed that the fluctuations of SpO2, StO2 and HR were synchronized in the low-frequency range with periods of $1 \mathrm{~h}$ and $0.5 \mathrm{~h}$. The amplitudes of the synchronization frequencies were dependent on the individual neonate. DISCUSSION: MECWA is a useful novel tool to assess the coupling of physiological signals. The parameters determined by MECWA seem to be related to the chronobiological processes, as well as constant regulations of the cardiovascular and cerebral perfusion state. CONCLUSION: MECWA was able to identify long-term synchronization of the cardiovascular and cerebral perfusion state in preterm neonates with periods of $1 \mathrm{~h}$ and $0.5 \mathrm{~h}$.
\end{abstract}

DOI: https://doi.org/10.1007/978-3-319-91287-5_25

Posted at the Zurich Open Repository and Archive, University of Zurich

ZORA URL: https://doi.org/10.5167/uzh-157689

Book Section

Accepted Version

Originally published at:

Scholkmann, Felix; Herrera, Graciela Velasco; Karen, Tanja; Isler, Helene; Ostojic, Daniel; Herrera, Victor Manuel Velasco; Wolf, Ursula; Wolf, Martin (2018). Synchronized Oscillations of Arterial Oxygen Saturation, Cerebral Tissue Oxygenation and Heart Rate in Preterm Neonates: Investigation of LongTerm Measurements with Multiple Einstein's Cross Wavelet Analysis. In: Thews, Oliver. Oxygen Transport to Tissue XL. Cham: Springer, 157-161.

DOI: https://doi.org/10.1007/978-3-319-91287-5_25 


\title{
Post-print version
}

Original publication: Scholkmann, F., Velasco Herrera, G., Karen, T., Isler, H., Ostojic, D. Velasco Herrera, V.M., Wolf, U. \& Wolf, M. (2018). Synchronized oscillations of arterial oxygen saturation, cerebral tissue oxygenation and heart rate in preterm neonates: Investigation of long-term measurements with multiple Einstein's cross wavelet analysis. Advances in Experimental Medicine and Biology, 1072, 157-161

\section{Synchronized oscillations of arterial oxygen saturation, cerebral tissue oxygenation and heart rate in preterm neonates: Investigation of long-term measurements with multiple Einstein's cross wavelet analysis}

Felix Scholkmann, Graciela Velasco Herrera, Tanja Karen, Helene Isler, Daniel Ostojic, Victor Manuel Velasco Herrera, Ursula Wolf, and Martin Wolf

\section{F. Scholkmann $(*)$}

Biomedical Optics Research Laboratory (BORL), Department of Neonatology, University Hospital Zurich, University of Zurich, Zurich, Switzerland Institute of Complementary Medicine, University of Bern, Bern, Switzerland, e-mail: Felix.Scholkmann@usz.ch

T. Karen · H. Isler · D. Ostojic · M. Wolf

Biomedical Optics Research Laboratory (BORL), Department of Neonatology, University Hospital Zurich, University of Zurich, Zurich, Switzerland

G. V. Herrera • V. M. V. Herrera

Universidad Nacional Autónoma de México, Ciudad Universitaria, Coyoacán, Mexico U. Wolf Institute of Complementary Medicine, University of Bern, Bern, Switzerland

\begin{abstract}
Background: In preterm neonates, the cardiovascular and cerebral vascular control is immature, making the brain vulnerable to an increased incidence of hypoxic and hyperoxic episodes. Aim: The aim of the study was to apply the recently developed multiple Einstein's cross wavelet analysis (MECWA) to quantify the coupling of fluctuations of peripherally measured arterial oxygen saturation $\left(\mathrm{SpO}_{2}\right)$, cerebral tissue oxygen saturation $\left(\mathrm{StO}_{2}\right)$ and heart rate $(\mathrm{HR})$. Methods: Two long-term measurements on preterm neonates with a gestational age at birth of 26.4 and 26.8 weeks and a postnatal age of 2.1 and 3.9 weeks were analyzed. MECWA was applied to $\mathrm{SpO}_{2}, \mathrm{StO}_{2}$ and HR. Results: MECWA showed that the fluctuations of $\mathrm{SpO}_{2}, \mathrm{StO}_{2}$ and $\mathrm{HR}$ were synchronized in the low-frequency range with periods of $\sim 1 \mathrm{~h}$ and $\sim 0.5 \mathrm{~h}$. The amplitudes of the synchronization frequencies were dependent on the individual neonate. Discussion: MECWA is a useful novel tool to assess the coupling of physiological signals. The parameters determined by MECWA seem to be related to the chronobiological processes, as well as constant regulations of the cardiovascular and cerebral perfusion state. Conclusion: MECWA was able to identify long-term synchronization of the cardiovascular and cerebral perfusion state in preterm neonates with periods of $\sim 1 \mathrm{~h}$ and $\sim 0.5 \mathrm{~h}$.
\end{abstract}




\section{Introduction}

Due to the immature cardiovascular and cerebral vascular system in preterm neonates, fluctuations in arterial oxygen saturation $\left(\mathrm{SpO}_{2}\right)$ and cerebral tissue oxygenation $\left(\mathrm{StO}_{2}\right)$ can lead to episodes of intermittent hypoxia and hyperoxia, resulting in a high risk of brain injuries with subsequent neurological morbidity and mortality [1-5].

Long-term (i.e., several hours) monitoring of changes in $\mathrm{SpO}_{2}$ and $\mathrm{StO}_{2}$ offers insights into tissue oxygenation and the regulation of hemodynamics. In a previous study in preterm neonates [6] we found that the fluctuations in $\mathrm{SpO}_{2}, \mathrm{SaO}_{2}$, heart rate (HR) and fractional tissue-oxygen extraction, measured over several hours, showed properties (i.e. coupling of the signals and oscillations present) dependent on the individual subject.

The aim of our study was to apply a novel signal processing method to these signals in order to analyze the coupling of them. With this study, we aimed to obtain novel insights into properties of cerebral and systemic hemodynamic fluctuations and their interdependences in preterm neonates.

\section{Data and Methods}

\subsection{Subjects, Instrumentation and Experimental Protocol}

Two long-term measurements on preterm neonates from a dataset of 20 single measurements (conducted during the night, i.e., from $\sim 10 \mathrm{pm}$ to $\sim 6 \mathrm{am}$ ) were selected for the analysis. The selection criterion was high data quality, i.e., few artifacts and gaps. The measurements were performed on two preterms with a gestational age of 26.4 and 26.8 weeks and a postnatal age of 2.1 and 3.9 weeks (for further details about the neonates, see Table 1). The measurements were approved by the local ethics committee, and written informed consent was obtained from the parents before the study. The datasets used are the same as those analyzed by our previous study investigating another signal processing and data analysis framework [6].

Absolute values of $\mathrm{StO}_{2}$ were measured over the left prefrontal cortex (PFC) employing an in-house developed multi-distance near-infrared spectroscopy (NIRS) oximeter (OxyPrem, $4 \times 3$ [760, 805, $870 \mathrm{~nm}$ ] light sources, two source-detector distances, [1.5 and $2.5 \mathrm{~cm}$ ]; calculation of $\mathrm{StO}_{2}$ based on the self-calibrating approach) [7]. HR and Spo2 were measured by a standard patient monitor (Infinity Delta XL, Dräger, Germany).

Table 1 Physiological parameters of the two preterm neoantes

\begin{tabular}{|c|c|c|}
\hline Parameters & $\begin{array}{l}\text { Preterm } \\
\text { neonate \#1 }\end{array}$ & $\begin{array}{l}\text { Preterm } \\
\text { neonate \#2 }\end{array}$ \\
\hline $\begin{array}{l}\text { GA at birth [weeks], GA } \\
\text { at measurement [weeks] }\end{array}$ & $26.4,28.5$ & $26.8,30.7$ \\
\hline Weight at measurement [g] & 1280 & 1440 \\
\hline Apgar $(1,5,10)$ & $5,4,5$ & $5,8,8$ \\
\hline Respiration & SIMV & spontaneous \\
\hline $\begin{array}{l}\mathrm{FiO}_{2}[\%], \mathrm{Hct}[\%], \mathrm{Hb}[\mathrm{g} / \\
\mathrm{dL}]\end{array}$ & $\begin{array}{l}25,40.9 \\
13.4\end{array}$ & $21,36,11.7$ \\
\hline $\begin{array}{l}\text { Length of analyzed data } \\
\text { [min] }\end{array}$ & 271 & 308 \\
\hline
\end{tabular}

$\mathrm{GA}$ gestational age, $\mathrm{FiO}_{2}$ fraction of inspired oxygen, SIMV synchronized intermittent mandatory ventilation, $\mathrm{Hct}$ hematocrit, $\mathrm{Hb}$ hemoglobin 


\subsection{Signal Processing and Data Analysis}

Since only low-frequency fluctuations were of interest for our study, and to increase the signal-to- noise- ratio (SNR), all signals $\left(\mathrm{SpO}_{2}, \mathrm{StO}_{2}, \mathrm{HR}\right)$ were first down sampled to $0.05 \mathrm{~Hz}$ and an additional low-pass filter was applied (locally weighted scatterplot smoothing, window length: 300 s). For each of the two datasets, a specific interval was chosen for the subsequent analysis containing only data without any signal distortions (271 min and $308 \mathrm{~min}$, respectively).

Multiple Einstein's cross wavelet analysis (MECWA) was applied to both datasets with the three input signals $\mathrm{SpO}_{2}, \mathrm{StO}_{2}$ and $\mathrm{HR}$ to quantify the coupling of fluctuations. The signals were normalized ( $z$-score) and linearly detrended before. MECWA is a recently developed specific type of cross wavelet transform [8] that uses a cross function originally proposed by Einstein [9] and generalizes the concept so that more than two signals can serve as an input. MECWA enables to analyze the time-frequency coupling of the signals with the multiple Einstein's cross wavelet power (MECWP) representing the main characteristic of the coupling. The main advantage of MECWA compared to a conventional Wavelet analysis is that MECWA allows having more than two input signals.
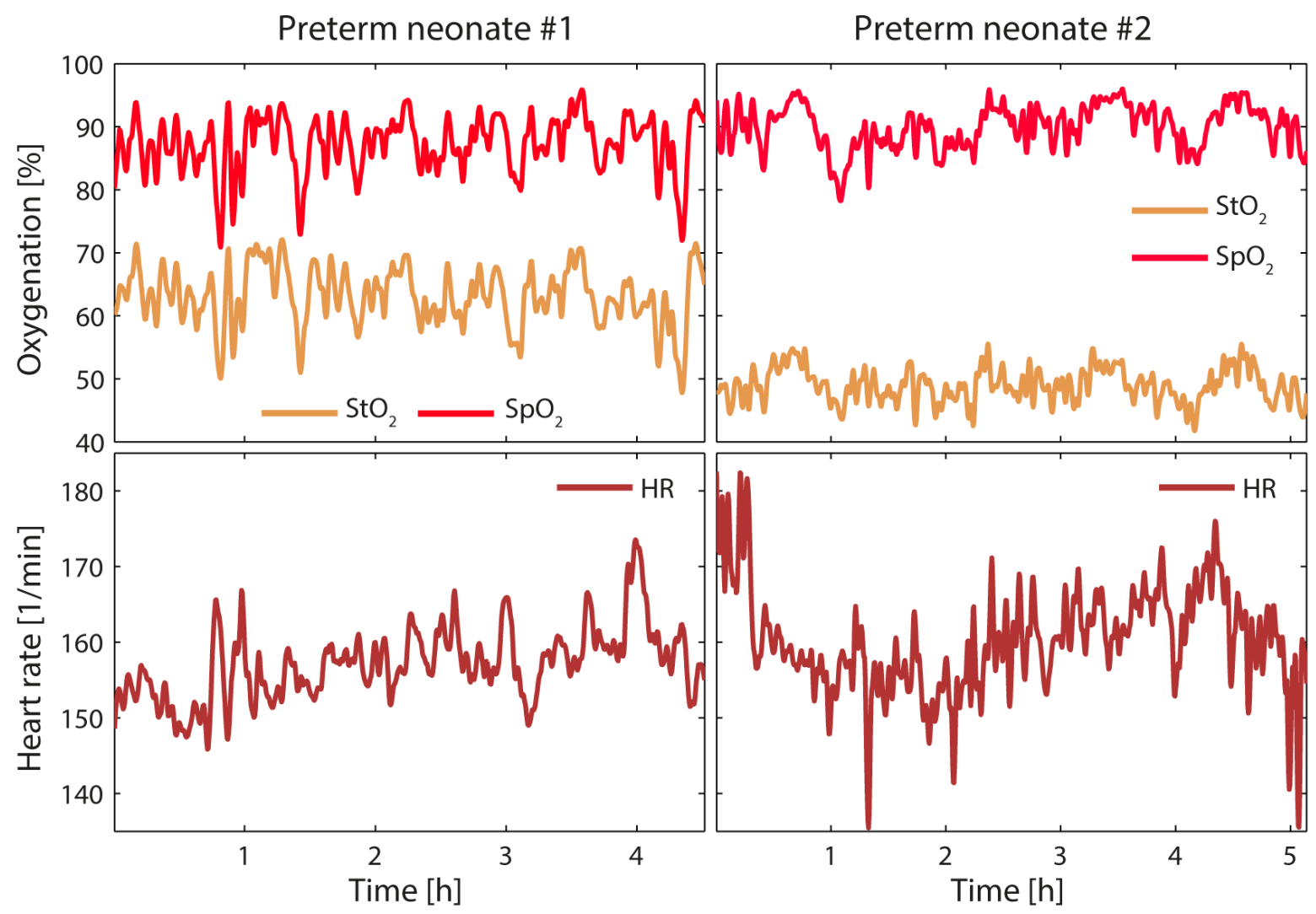

Fig 1. Visualization of the analyzed signals $\left(\mathrm{StO}_{2}, \mathrm{SpO}_{2}\right.$ and $\left.\mathrm{HR}\right)$ of both data sets.

\section{Results, Discussion, Conclusion and Outlook}

The $\mathrm{SpO}_{2}, \mathrm{StO}_{2}$ and $\mathrm{HR}$ signals from the two datasets used for the analysis are depicted in Fig. 1. The results of the MECWA are shown in Fig. 2. The results can be summarized as follows: 
- Both preterm neonates showed a coupling of $\mathrm{SpO}_{2}, \mathrm{StO}_{2}$ and $\mathrm{HR}$ in the low-frequency range with periods of $\sim 1 \mathrm{~h}$ and $\sim 0.5 \mathrm{~h}$.

- The amplitudes of the coupling were dependent on the individual neonate: preterm \#1 had a strong coupling with an oscillatory period $(\tau)$ of $0.5483 \mathrm{~h}$ and a weaker one (i.e., $49.9 \%$ of the power of that of the first one) with $\tau=0.87038 \mathrm{~h}$. In preterm \#2 this trend was reversed: the lower frequency oscillation of coupling $(\tau=1.031 \mathrm{~h})$ was stronger than the higher-frequency one $(\tau=0.54617 \mathrm{~h})$. The latter was only $0.94 \%$ of the power of the first one.

How can these results be interpreted physiologically? First, the results indicate that spontaneous long-term changes in cerebral and systemic hemodynamics/oxygenation $\left(\mathrm{StO}_{2}\right.$ vs. $\mathrm{SpO}_{2}$ and $\mathrm{HR}$ ) are synchronized to each other. Second, this synchronization is in a specific frequency range with periods of $\sim 1 \mathrm{~h}$ and $\sim 0.5 \mathrm{~h}$. It is known that neonates exhibit a sleep-wake cycle in this frequency range $(\tau=50-60 \mathrm{~min}$, quiet sleep phase duration: $\sim 20$ min) [10]. A change in HR during the sleep cycle (quiet vs. active sleep) was also reported to be present in neonates [11, 12]. The synchronization frequencies observed in our Fig. 1 study match well with these frequencies, indicating that the cerebral-systemic hemodynamic state is modulated by the sleep-wake cycle or by changes in the sleep state. The long-term oscillations observed seem thus to be related to chronobiological processes.

The reason why preterm neonate \#1 showed a modulation coupling with a higher frequency than preterm neoante \#2 is not directly obvious. Many reasons could account for this. Whereas the GA as well as weight, $\mathrm{FiO}_{2}$, $\mathrm{Hct}$ and $\mathrm{Hb}$ were similar in both neonates, the type of respiration was different: preterm neonate \#1 was on mechanical ventilation (SIMV) whereas preterm neonate \#2 was breathing spontaneously.

In conclusion, using long-term measurements on two preterm neonates, we demonstrated the applicability of a novel signal-processing method, MECWA, to investigate the coupling of cerebral and systemic hemodynamic signals in the timefrequency space. A limitation of our study is that only data from two neonates are reported in the present manuscript. More data sets are available and will be analyzed in the future.

Low-frequency oscillations in the coupling were observed, indicating that the cerebral and systemic hemodynamic state is especially synchronized in the low-frequency region (i.e., with period lengths of $>20 \mathrm{~min}$ ). As already indicated in our previous publication [6], analysis of spontaneous fluctuations of cerebral and systemic hemodynamic signals and their synchronization offer novel insights into the physiological state of the neonate investigated which are not assessed routinely. The present approach could be extended by also adding the arterial carbon dioxide pressure $\left(\mathrm{PaCO}_{2}\right)$ signal to the input signals for MECWA since fluctuations in $\mathrm{PaCO}_{2}$ are also relevant for hemodynamic state in preterm neonates [13]. To the best of our knowledge, this is the first application of MECWA to human NIRS data. 

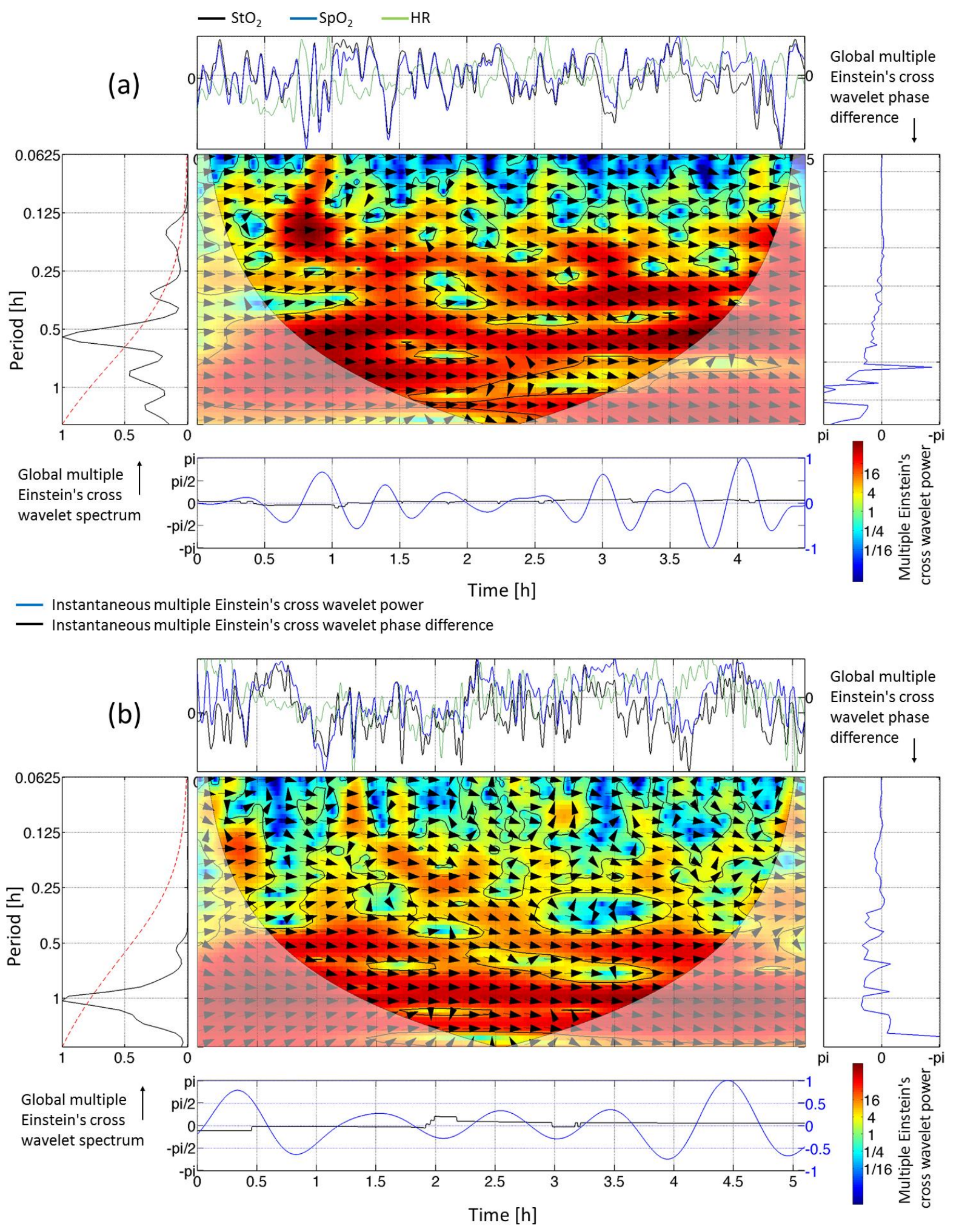

Fig. 2 Visualization of the MECWA results for the two datasets. (a) dataset 1 (preterm neonate\#1), (b) dataset 2 (preterm neonate \#2).

\section{Acknowledgments}

We gratefully acknowledge funding by Nano-Tera (NeoSense, ObeSense), the Swiss National Science Foundation, the Neuroscience Center Zurich (UCL-Zurich Collaboration), The Danish Council for Strategic Resarch (SafeBoosC). 


\section{References}

1. Stammwitz A, von Siebenthal K, Bucher HU et al (2016) Can the assessment of spontaneous oscillations by near infrared spectrophotometry predict neurological outcome of preterm infants? Adv Exp Med Biol 876:521-531

2. Fyfe KL, Yiallourou SR, Wong FY et al (2014) The development of cardiovascular and cerebral vascular control in preterm infants. Sleep Med Rev 18(4):299-310

3. Brew N, Walker D, Wong FY (2014) Cerebral vascular regulation and brain injury in preterm infants. Am J Physiol Regul Integr Comp Physiol 306(11):R773-R786

4. Martin RJ, Wang K, Koroglu O et al (2011) Intermittent hypoxic episodes in preterm infants: do they matter? Neonatology 100(3):303-310

5. Horne RS, Fung AC, NcNeil S et al (2017) The longitudinal effects of persistent apnea on cerebral oxygenation in infants born preterm. J Pediatr 182:79-84

6. Kleiser S, Pastewski M, Hapuarachchi T et al (2016) Characterizing fluctuations of arterial and cerebral tissue oxygenation in preterm neonates by means of data analysis techniques for nonlinear dynamical systems. Adv Exp Med Biol 876:511-519

7. Hyttel-Sorensen S, Kleiser S, Wolf M et al (2013) Calibration of a prototype NIRS oximeter against two commercial devices on a blood-lipid phantom. Biomed Opt Express 4(9):1662-1672

8. Velasco Herrera VM, Soon W, Velasco Herrera G et al (2017) Generalization of the crosswavelet function. New Astron 56:86-93

9. Einstein A (1914) Method for the determinination of the statistical values of observations concerning quantities subject to irregular fluctuations. Arch Sci Phys Nat 37:254-256

10. Osredkar D, Toet MC, van Rooij LG et al (2005) Sleep-wake cycling on amplitude-integrated electroencephalography in term newborns with hypoxic-ischemic encephalopathy. Pediatrics 115(2): 327-332

11. Münger DM, Bucher HU, Duc G (1998) Sleep state changes associated with cerebral blood volume changes in healthy term newborn infants. Early Hum Dev 52(1):27-42

12. Stéphan-Blanchard E, Chardon K, Léké A et al (2013) Heart rate variability in sleeping preterm neonates exposed to cool and warm thermal conditions. PLoS One 8(7):e68211

13. Fabres J, Carlo WA, Phillips V et al (2007) Both extremes of arterial carbon dioxide pressure and the magnitude of fluctuations in arterial carbon dioxide pressure are associated with severe intraventricular hemorrhage in preterm infants. Pediatrics 119(2):299-305 